Non-ruminants

\title{
Creep feeding improves growth performance of suckling piglets
}

\author{
Sang In Lee ${ }^{1}$, In Ho Kim ${ }^{1 *}$ \\ ${ }^{1}$ Dankook University, Department of Animal Resource and Science, Cheonan, Choongnam, Republic of Korea.
}

ABSTRACT - The present study evaluated the optimal duration of creep feeding that improves the growth performance and fecal score of piglets and the performance and estrus interval of sows. Sixteen sows (Yorkshire $\times$ Landrace) and their litters were used in this 24-day trial. Dietary treatments were: Treatment 1 , in which creep feed was not provided; treatment 2 , in which creep feed was provided at the 7 th day of life (day 7 to weaning); treatment 3 , in which creep feed was provided at the 14th day of life (day 14 to weaning); and treatment 4, in which creep feed was provided at the 21 st day of life (day 21 to weaning). There was no difference in body weight or loss, backfat thickness or loss, or estrus interval among treatments in the sows. Piglets in treatment 2 had higher body weight (BW) than pigs of treatments 1, 3, and 4 . In addition, piglets in treatment 3 had higher BW than those of treatments 1 and 4. From birth to weaning, piglets of treatments 2 and 3 had higher average daily gain than those of treatments 1 and 4 . There was no difference in fecal score of piglets among treatments. Piglets fed creep feed initiated from the first week have higher BW and average daily gain at weaning compared with those initiated from the second and third weeks, indicating that a longer duration of creep feeding improves the growth performance of piglets.

Key Words: creep feeding, growth performance, sow, suckling piglets

\section{Introduction}

The birth and weaning weights are critically important in the life of pigs because the weight of piglets at birth and weaning determines their post-weaning performance and the time needed to reach market weight (Mahan, 1993). Many researchers have tried to increase pig weight during preweaning by using a sow feeding program during the lactation period (Kim et al., 2008; Plante et al., 2011; Walsh et al., 2012; Sun et al., 2015). In addition to sow feeding programs during lactation, piglet feeding programs such as milk replacers and creep feed have been used to increase weight during pre-weaning (Yan et al., 2011a, 2011b, 2011c).

Creep feed has been used to fill the gap between the increasing nutrient requirements of suckling pigs and the nutrients supplied by the lactating sow and to adapt feedstuffs after weaning. It has been reported that creep feeding improves the post-weaning growth performance of piglets (Pajor et al., 1991; Yan et al., 2011b). In addition, creep feeding influences reproductive performance of

Received: May 1, 2017

Accepted: October 12, 2017

*Corresponding author: inhokim@dankook.ac.kr

Copyright (C) 2018 Sociedade Brasileira de Zootecnia. This is an Open Access article distributed under the terms of the Creative Commons Attribution License (http://creativecommons.org/licenses/by/4.0/), which permits unrestricted use, distribution, and reproduction in any medium, provided the original work is properly cited. piglets, nutritional load, body weight (BW), as well as the weaning-to-estrus interval of lactating sows (Foxcroft, 1992).

The duration of creep feeding is important for the performance of piglets and sows (Klindt, 2003; Sulabo et al., 2010). It has been reported that creep feeding two weeks before weaning resulted in greater pre-weaning daily gains than did creep feeding two days before weaning in piglets (Klindt, 2003). However, in another report, longer durations of creep feeding increased feed intake, but did not affect growth performance during pre-weaning (Sulabo et al., 2010). There is a limited amount of evidence on the effect of the optimal duration of creep feeding on the growth performance, fecal score of piglets, performance, and estrus interval of lactating sows. Thus, in the present study, we evaluated the optimal duration of creep feeding in the improvement of growth performance and fecal score of piglets, as well as the performance and estrus interval of sows.

\section{Material and Methods}

The animal care and protocol used in this study were approved by the Animal Care and Use Committee (ACUCDU1302406).

Sixteen sows (Yorkshire $\times$ Landrace) and their litters were used in this 24-day trial. Sows were assigned randomly to one of four creep feeding groups, with average parities of 
$3.2 \pm 0.5$. At day 107 of gestation, the sows were moved to farrowing crates in an environmentally regulated farrowing house. Dietary treatment and feed were as follows: treatment 1 , in which creep feed was not provided; treatment 2 , in which creep feed was provided at the 7th day of life (day 7 to weaning); treatment 3 , in which creep feed was provided at the 14th day of life (day 14 to weaning); and treatment 4 , in which creep feed was provided at the 21 st day of life (day 21 to weaning). Sows were fed a lactation feed program that increased to $7 \mathrm{~kg}$ and the weaning of piglets occurred at day 24 (Table 1).

Sows and their offspring were individually housed in farrowing crates $(2.4 \times 1.8 \mathrm{~m})$ that were constructed with $1.95 \mathrm{~m}^{2}$ of solid floor and $2.37 \mathrm{~m}^{2}$ of slatted floor. This space included a piglet nest equipped with an infrared lamp $(500 \mathrm{~W})$, a piglet drinking nipple, and a piglet feeder placed on dimpled rubber matting to collect any spillage from the feed. The temperature in the farrowing house was maintained at a minimum of $20{ }^{\circ} \mathrm{C}$. Drinking nipples provided water ad libitum to both sows and the piglets. All piglets received injections of $1 \mathrm{~mL}$ of iron dextran and the males were castrated two days after birth. At weaning, the sows were relocated to a mating room, with the piglets remaining in the pen for three days; the number of weanling piglets was recorded.

Individual piglet BW was obtained on days $0,7,14$, 21, and 24 at weaning, and on days 7, 14, 21, and 24 after birth to calculate the average daily gain (ADG). Creep feed residuals and general health was checked daily. Sow body weights were checked within a few hours after farrowing and on the day of weaning, after which the backfat thickness of the sows (6 $\mathrm{cm}$ off the midline at the 10th rib) was measured using a real-time ultrasound instrument (Herlev, Denmark).

Fecal score in piglets was observed and recorded three times per day throughout the study. To assess the fecal score, feces from each pig was scored by estimating the moisture content (Hart and Dobb, 1988). In brief, the scores were as follows: 1 , hard and dry pellet; 2 , firmly formed stool; 3 , soft and moist stool that retains shape; 4 , soft and unformed stool that assumes the shape of the container; and 5, watery liquid that can be poured. A cumulative fecal score per diet and day was then calculated (Montagne et al., 2004). Fecal scores of piglets were recorded at days $7,14,21$, and at weaning.

Table 1 - Composition of experimental diets (as-fed basis)

\begin{tabular}{|c|c|c|c|}
\hline \multicolumn{2}{|c|}{ Diet for sows } & \multicolumn{2}{|c|}{ Diet for piglets } \\
\hline Ingredient (\%) & & Ingredient (\%) & \\
\hline Corn & 51.00 & Corn & 22.62 \\
\hline Soybean meal $(48 \% \mathrm{CP})$ & 26.73 & Soybean meal $(48 \% \mathrm{CP})$ & 8.00 \\
\hline Wheat bran & 1.00 & Soy oil & 4.50 \\
\hline Rice bran & 5.00 & Whey & 24.16 \\
\hline Rapeseed meal (43\% CP) & 3.50 & Fish meal & 2.50 \\
\hline Tallow & 6.05 & Fermented soybean meal & 10.00 \\
\hline Molasses & 3.50 & Coconut oil & 4.17 \\
\hline Dicalcium phosphate & 1.64 & Lactose & 8.00 \\
\hline Limestone & 0.76 & Plasma powder & 4.00 \\
\hline Salt & 0.50 & Sugar & 3.05 \\
\hline L-lysine-HCl (78\%) & 0.12 & Isolated soybean protein & 6.15 \\
\hline Vitamin $^{1}$ & 0.10 & Dicalcium phosphate & 1.25 \\
\hline Mineral $^{2}$ & 0.10 & DL-methionine & 0.38 \\
\hline Total & 100 & L-lysine $\cdot \mathrm{HCl}(78 \%)$ & 0.41 \\
\hline \multirow{2}{*}{\multicolumn{2}{|c|}{ Calculated composition (\%) }} & Threonine (98\%) & 0.13 \\
\hline & & Zinc oxide & 0.30 \\
\hline Metabolizable energy $(\mathrm{kcal} / \mathrm{kg})$ & 3,456 & Choline $\mathrm{Cl}(50 \%)$ & 0.10 \\
\hline Crude protein & 18.34 & Vitamin $^{1}$ & 0.10 \\
\hline Crude fat & 9.16 & Mineral $^{2}$ & 0.18 \\
\hline Lysine & 1.08 & Total & 100.00 \\
\hline Calcium & 1.06 & & 100.00 \\
\hline \multirow[t]{7}{*}{ Phosphorus } & 0.73 & Calculated composition (\%) & \\
\hline & & Digestible energy $(\mathrm{kcal} / \mathrm{kg})$ & 4,000 \\
\hline & & Crude protein & 22.00 \\
\hline & & Lysine & 1.74 \\
\hline & & Methionine & 0.70 \\
\hline & & Calcium & 0.81 \\
\hline & & Phosphorus & 1.00 \\
\hline
\end{tabular}

\footnotetext{
CP - crude protein.
}

${ }^{1}$ Provided per kg of complete diet: vitamin A, 11,025 IU; vitamin D3, 1,103 IU; vitamin E, 44 IU; vitamin K, 4.4 mg; riboflavin, 8.3 mg; niacin, 50 mg; thiamine, 4 mg; pantothenic acid, $29 \mathrm{mg}$; choline, $166 \mathrm{mg}$; and vitamin B12, $33 \mu \mathrm{g}$.

${ }^{2}$ Provided per kg of complete diet: $\mathrm{Cu}, 12 \mathrm{mg}$; Zn, $85 \mathrm{mg}$; Mn, $8 \mathrm{mg}$; I, $0.28 \mathrm{mg}$; and Se, $0.15 \mathrm{mg}$. 
Detection of estrus was conducted twice per day from weaning onward. A sow was considered in estrus when it exhibited a standing response induced by a backpressure test in the presence of a boar.

All data in this experiment were analyzed in accordance with a completely randomized design using the GLM procedure of SAS (Statistical Analysis System, version 9.4). The individual sow or litter of piglets was used as the experimental unit. Differences among treatment means were determined via Duncan's multiple range tests regarded as significant at a probability level of $\mathrm{P}<0.05$.

\section{Results}

There was no difference in BW or BW loss, backfat thickness or backfat loss, or estrus interval of sows among treatments $(\mathrm{P}>0.05$; Table 2$)$. No difference was observed in $\mathrm{BW}$ initially and at one week ( $\mathrm{P}>0.05$; Figure 1$)$. Piglets in treatment 2 had higher BW than those in treatments 1,3 , and 4 at the second week $(\mathrm{P}<0.05)$. At the third week and weaning, piglets in treatment 3 had higher BW than those in treatments 1 and 4; piglets in treatment 2 had higher $\mathrm{BW}$ than pigs in treatments 1,3 , and $4(\mathrm{P}<0.05)$. No difference was observed in ADG at the first week $(\mathrm{P}>0.05)$. Piglets in treatment 2 had higher ADG than those in treatments 1, 3, and 4 at the second week $(\mathrm{P}<0.05)$. Diets of treatments 2 and 3 resulted in higher ADG than treatments 1 and 4 at the third week $(\mathrm{P}<0.05)$. At weaning, piglets in treatments 2, 3 ,

Table 2 - Effects of different initiation of creep feeding on growth performance of sows

\begin{tabular}{|c|c|c|c|c|c|}
\hline \multirow{2}{*}{ Item } & \multicolumn{4}{|c|}{ Treatment $^{1}$} & \multirow{2}{*}{ SEM } \\
\hline & 1 & 2 & 3 & 4 & \\
\hline \multicolumn{6}{|l|}{ Body weight (kg) } \\
\hline Before farrowing & 224.35 & 221.70 & 236.53 & 243.03 & 8.108 \\
\hline After farrowing & 197.68 & 201.15 & 217.93 & 213.25 & 8.723 \\
\hline After weaning & 175.65 & 177.33 & 195.05 & 185.90 & 10.077 \\
\hline \multicolumn{6}{|l|}{ Body weight loss (kg) } \\
\hline After farrowing & 26.68 & 20.55 & 18.60 & 29.78 & 3.422 \\
\hline After weaning & 22.03 & 23.83 & 22.88 & 27.35 & 2.034 \\
\hline \multicolumn{6}{|c|}{ Average daily feed intake $(\mathrm{kg})$} \\
\hline Farrowing & 1.07 & 0.96 & 1.02 & 1.06 & 0.154 \\
\hline Farrowing to weaning & 6.86 & 6.84 & 6.82 & 6.89 & 0.036 \\
\hline \multicolumn{6}{|l|}{ Backfat thickness (mm) } \\
\hline Before farrowing & 20.92 & 17.17 & 17.34 & 18.92 & 2.237 \\
\hline After farrowing & 18.42 & 16.67 & 16.66 & 18.33 & 1.068 \\
\hline After weaning & 11.75 & 11.84 & 13.08 & 12.83 & 0.802 \\
\hline \multicolumn{6}{|l|}{ Backfat loss (mm) } \\
\hline After weaning & 6.67 & 4.83 & 3.58 & 5.50 & 0.946 \\
\hline Estrus interval (days) & 4.75 & 4.50 & 4.50 & 4.75 & 0.270 \\
\hline
\end{tabular}

and 4 had higher ADG than those in treatment $1(\mathrm{P}<0.05)$. From birth to weaning, piglets in treatments 2 and 3 had higher ADG than those in treatments 1 and $4(\mathrm{P}<0.05)$. There were no differences in birth and weaning number, still birth and piglet survival rate, or fecal score of piglets among treatments (Table 3).

\section{Discussion}

Weaning weight is important for determining postweaning performance, such as weight at the final age and yield of marketable pork (Mahan, 1993). A number of studies have been performed to improve the weight of piglets at weaning, focusing on nutrition during gestation and gestation of sows and suckling piglets (Yan et al., 2011a, 2011b, 2011c). Focusing on piglet nutrition, Heo et al. (1999) demonstrated that a milk replacer feeding system utilized in the early suckling period can maximize pig growth performance and that ADG, average daily feed intake, and gain-to-feed ratio were affected by different ambient temperatures within milk replacer-fed pigs. In the present study, we focused on the nutrition of suckling piglets to improve growth performance from birth to weaning by using creep feeding.

It is known that creep feeding can improve growth performance during the suckling period by filling the gap between the increasing nutrient requirement of suckling pigs and the nutrients supplied by the lactating sow; it has also been used to adapt feedstuffs after weaning of suckling piglets (Pajor et al., 1991; Sulabo et al., 2010; Yan et al., 2011a, 2011b, 2011c). A number of reports has focused

Table 3 - Effects of different initiation of creep feeding on birth and weaning number, still birth and piglet survival rate, and fecal score of piglets

\begin{tabular}{lccccc}
\hline \multirow{2}{*}{ Item } & \multicolumn{5}{c}{ Treatment $^{1}$} \\
\cline { 2 - 5 } & 1 & 2 & 3 & 4 & SEM \\
\hline Birth (n) & 11.75 & 10.25 & 10.5 & 12.25 & 1.11 \\
Weaning (n) & 10.75 & 9.5 & 9.75 & 10.5 & 1.19 \\
Still birth (\%) & 0 & 0 & 1 & 0.5 & 0.38 \\
Piglet survival (\%) & 92.72 & 92.22 & 89.58 & 87.09 & 6.48 \\
Total creep feed intake (kg) & & $7.49 \mathrm{a}$ & $4.82 \mathrm{~b}$ & $1.56 \mathrm{c}$ & 0.67 \\
Fecal score & & & & & \\
$\quad$ 1st week & 4.3 & 4.9 & 4.1 & 4.4 & 0.66 \\
2nd week & 3 & 3 & 3 & 3 & 0.00 \\
3rd week & 3 & 3 & 3 & 3 & 0.00 \\
$\quad$ Weaning & 3 & 3 & 3 & 3 & 0.00 \\
\hline
\end{tabular}

SEM - standard error of mean.

${ }^{1}$ Treatment 1: creep feed was not provided; treatment 2: creep feed was provided from seven days to weaning; treatment 3 : creep feed was provided from 14 days to weaning; treatment 4: creep feed was provided from 21 days to weaning.

${ }^{2}$ The scores were as follows: 1 , hard and dry pellet; 2 , firmly formed stool; 3 , soft and moist stool that retains shape; 4 , soft and unformed stool that assumes the shape of the container; and 5, watery liquid that can be poured.

a,b,c - Within a row, means with different letters differ $(\mathrm{P}<0.05)$. 
A

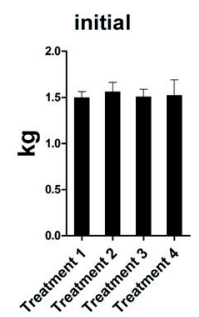

B
Body weight

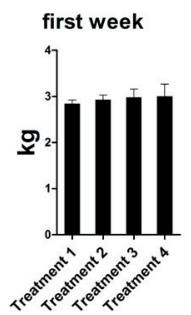

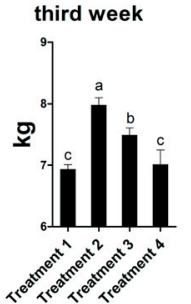

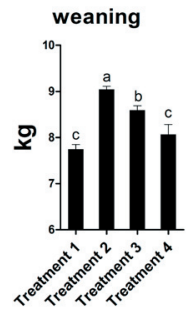

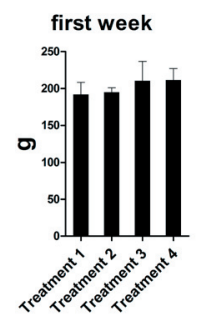

Average daily gain
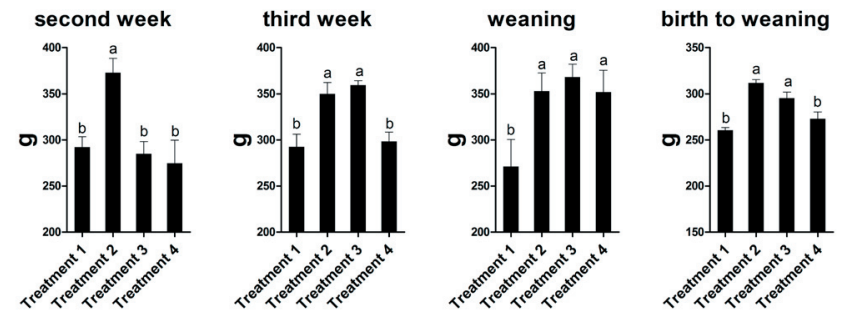

A - body weight; B - average daily gain.

Treatment 1: creep feed was not provided; treatment 2: creep feed was provided from 7 days to weaning; treatment 3: creep feed was provided from 14 days to weaning; treatment 4: creep feed was provided from 21 days to weaning.

Differences among treatment means were determined using the Duncan's multiple range tests. Statements of statistical significance were based on $\mathrm{P}<0.05$.

Error bars indicate the standard error of analyses $(n=40)$.

a,b,c - Within a row, means with different letters differ $(\mathrm{P}<0.05)$.

Figure 1 - Effect of creep feeding on growth performance.

on the duration of creep feeding to improve the growth performance of suckling piglets (Klindt, 2003; Sulabo et al., 2010; Yan et al., 2011c). Klindt (2003) demonstrated that creep feeding two weeks before weaning resulted in greater pre-weaning daily gains of piglets compared with creep feeding two days before weaning. Sulabo et al. (2010) demonstrated that longer durations of creep feeding increased feed intake, but did not show positive effects on growth performance during suckling. In another report, varying the duration of creep feeding had no effect on the growth performance of pre- and postweaning piglets (Yan et al., 2011c). From these reports, we hypothesized that the duration of creep feeding is important for improving the growth performance of suckling piglets. In addition, there is a limited amount of evidence regarding the effect of the optimal initiation of creep feeding on the growth performance of suckling piglets. Thus, in the present study, we evaluated the optimal initiation of creep feeding to improve the growth performance and fecal score of piglets, as well as the performance and estrus interval of sows. In the present study, suckling piglets that were fed creep feed, initially provided seven days after birth, had higher BW and ADG than those fed 14 and 21 days after birth. We suggest that the early initiation of creep feeding can improve the growth performance of sucking piglets at weaning.
In this study, varying the initiation of creep feeding had no effect on growth performance, BW, average daily feed intake, backfat thickness and backfat loss, or estrus interval in sows. Inconsistent with the present study, Foxcroft (1992) reported that creep feeding in piglets influences the reproductive performance, nutritional load, $\mathrm{BW}$, and weaning-to-estrus interval of lactating sows. In addition, creep feeding in sows significantly reduced their weaning-to-estrus interval and concentrations of cortisol, epinephrine, and norepinephrine and did not affect their nursing, eating, standing times, backfat loss, or BW loss (Yan et al., 2011c). However, Yan et al. (2011a) reported that creep feeding made no difference in blood characteristics, backfat loss, estrus, or behavior of sows. To the best of our knowledge, studies on the effects of creep feeding on sow performance are somewhat inconsistent. Therefore, further study is required to clarify the effects of creep feeding on piglets and sows because there are no studies that can be compared to the present study.

In our previous study, creep feeding was initiated on days 5, 10, and 15 from birth to weaning (21 days) and varying the duration of creep feed had no effect on preweaning piglet growth performance or on suckling, sleeping, fighting, and mortality. In addition, creep feeding initiated from days 5 and 10 reduced the post-weaning diarrhea score. However, in disagreement with that study, 
creep feeding initiated on days 7 and 14 in the present study had positive effects on pre-weaning growth performance. Indeed, the effect of creep feeding on the pre-weaning growth performance of suckling pigs is inconsistent in previous studies (Klindt 2003; Sulabo et al., 2010; Yan et al., 2011b). Klindt (2003) suggested that creep feeding from five days of age resulted in greater pre-weaning daily gain than creep feeding from two days prior to weaning (17 days). However, Sulabo et al. (2010) demonstrated that varying creep feeding duration had no effect on preweaning gain and weaning weight. Even in our previous studies, different energy and duration of creep feeding did not affect the pre-weaning growth performance (Yan et al., 2011a, 2011b). The reason for this significant difference between our present and previous studies is unknown; it may have occurred due to seasonal effects and birth weight differences. The previous study evaluating different energy and durations of creep feeding was performed after farrowing in the summer, whereas the present study was performed in the winter. It is well accepted that the farrowing season is one of the most important environmental factors directly affecting reproductive performance, such as litter size, piglet survival after birth, and piglet birth weight (Peltoniemi and Virolainen, 2006; Knecht et al., 2015). In the present study, the $\mathrm{BW}$ of piglets was $1.52 \pm 0.11 \mathrm{~kg}$, which is higher than the $1.18 \pm 0.12 \mathrm{~kg}$ of the previous study. It has been reported that birth weight affects weight, growth performance, backfat, and longissimus muscle area from pre-weaning to slaughter (Skorjanc et al., 2007; Fix et al., 2010). Skorjanc et al. (2007) reported that high-birth-weight piglets had higher BW at weaning and low-birth-weight piglets had 17 to $22 \%$ lower ADG when compared with heavy and intermediatebirth-weight piglets. However, it is not sufficient to explain the difference in results for creep feeding in our studies. In addition to difference in birth weight according to season, the effect of creep feeding may be affected by many factors such as condition, parity, and the nutritional condition of sows and the birth weight of their litters and piglets. Thus, further investigation is necessary to evaluate the effect of creep feeding, considering the many factors that affect it.

\section{Conclusions}

Suckling piglets fed creep feed, initially provided seven days after birth, have higher body weight and average daily gain than those fed 14 and 21 days after birth. We suggest that a longer duration of creep feeding can improve the growth performance of sucking piglets at weaning.

\section{Acknowledgments}

The present research was conducted by the research fund of Dankook University in 2018.

\section{References}

Fix, J. S.; Cassady, J. P.; Herring, W. O.; Holl, J. W.; Culbertson, M. S. and See, M. T. 2010. Effect of piglet birth weight on body weight, growth, backfat, and longissimus muscle area of commercial market swine. Livestock Science 127:51-59.

Foxcroft, G. R. 1992. Nutritional and lactational regulation of fertility in sows. Journal of Reproduction and Fertility 45:113-125.

Hart, G. K. and Dobb, G. J. 1988. Effect of a fecal bulking agent on diarrhea during enteral feeding in the critically ill. Journal of Parenteral and Enteral Nutrition 12:465-468.

Heo, K. N.; Odle, J.; Oliver, W.; Kim, J. H.; Han, I. K. and Jones, E. 1999. Effects of milk replacer and ambient temperature on growth performance of 14-day-old early-weaned pigs. Asian-Australasian Journal of Animal Sciences 12:908-913.

Kim, S. W.; Brandherm, M.; Freeland, M.; Newton, B.; Cook, D. and Yoon, I. 2008. Effects of yeast culture supplementation to gestation and lactation diets on growth of nursing piglets. Asian-Australasian Journal of Animal Sciences 21:1011-1014.

Klindt, J. 2003. Influence of litter size and creep feeding on preweaning gain and influence of preweaning growth on growth to slaughter in barrows. Journal of Animal Science 81:2434-2439.

Knecht, D.; Srodon, S. and Duzinski, K. 2015. The impact of season, parity and breed on selected reproductive performance parameters of sows. Archiv fur Tierzucht 58:49-56.

Mahan, D. C 1993. Effect of weight, split-weaning, and nursery feeding programs on performance responses of pigs to 105 kilograms body weight and subsequent effects on sow rebreeding interval. Journal of Animal Science 71:1991-1995.

Montagne, L.; Cavaney, F. S.; Hampson, D. J.; Lalles, J. P. and Pluske, J. P. 2004. Effect of diet composition on postweaning colibacillosis in piglets. Journal of Animal Science 82:2364-2374.

Pajor, E. A.; Fraser, D. and Kramer, D. L. 1991. Consumption of solid food by suckling pigs: individual variation and relation to weight gain. Applied Animal Behaviour Science 32:139-155.

Peltoniemi, O. A. and Virolainen, J. V. 2006. Seasonality of reproduction in gilts and sows. Society of Reproduction and Fertility Supplement 62:205-218.

Plante, P. A.; Laforest, J. P. and Farmer, C. 2011. Effect of supplementing the diet of lactating sows with NuPro ${ }^{\circledR}$ on sow lactation performance and piglet growth. Canadian Journal of Animal Science 91:295-300.

Skorjanc, D.; Brus, M. and Potokar, M. C. 2007. Effect of birth weight and sex on pre-weaning growth rate of piglets. Archiv fur Tierzucht 50:476-486.

Sulabo, R. C.; Tokach, M. D.; Dritz, S. S.; Goodband, R. D.; de Rouchey, J. M. and Nelssen, J. L. 2010. Effects of varying creep feeding duration on the proportion of pigs consuming creep feed and neonatal pig performance. Journal of Animal Science 88:3154-3162

Sun, H. Q.; Tan, C. Q.; Wei, H. K.; Zou, Y.; Long, G.; Ao, J. T.; Xue, H. X.; Jiang, S. W. and Peng, J. 2015. Effects of different amounts of konjac flour inclusion in gestation diets on physio-chemical properties of diets, postprandial satiety in pregnant sows, lactation 
feed intake of sows and piglet performance. Animal Reproduction Science 152:55-64.

Walsh, M. C.; Geraert, P. A.; Maillard, R.; Kluess, J. and Lawlor, P. G. 2012. The effect of a non-starch polysaccharide-hydrolysing enzyme (Rovabio ${ }^{\circledR}$ Excel) on feed intake and body condition of sows during lactation and on progeny growth performance. Animal 6:1627-1633.

Yan, L.; Jang, H. D. and Kim, I. H. 2011a. Creep feed: Effects of feed flavor supplementation on pre-and post-weaning performance and behavior of piglet and sow. Asian-Australasian Journal of Animal Sciences 24:851-856.

Yan, L.; Jang, H. D. and Kim, I. H. 2011b. Effects of creep feed with varied energy density diets on litter performance. AsianAustralasian Journal of Animal Sciences 24:1435-1439.

Yan, L.; Jang, H. D. and Kim, I. H. 2011c. Effects of varying creep feed duration on pre-weaning and post-weaning performance and behavior of piglet and sow. Asian-Australasian Journal of Animal Sciences 24:1601-1606. 